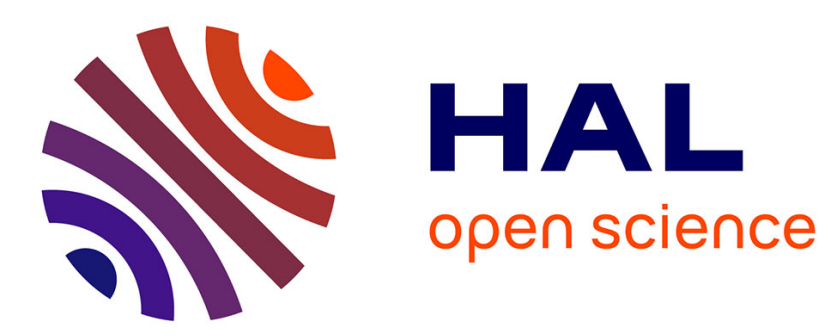

\title{
The motion of a compressible viscous fluid around rotating body
}

Stanislav Kracmar, Sarka Necasova, Antonin Novotny

\section{To cite this version:}

Stanislav Kracmar, Sarka Necasova, Antonin Novotny. The motion of a compressible viscous fluid around rotating body. Annali dell'Universita di Ferrara, 2014, 60 (1), pp.189-209. hal-00998361

\section{HAL Id: hal-00998361 \\ https://hal-univ-tln.archives-ouvertes.fr/hal-00998361}

Submitted on 1 Jun 2014

HAL is a multi-disciplinary open access archive for the deposit and dissemination of scientific research documents, whether they are published or not. The documents may come from teaching and research institutions in France or abroad, or from public or private research centers.
L'archive ouverte pluridisciplinaire HAL, est destinée au dépôt et à la diffusion de documents scientifiques de niveau recherche, publiés ou non, émanant des établissements d'enseignement et de recherche français ou étrangers, des laboratoires publics ou privés. 


\title{
The motion of a compressible viscous fluid around rotating body
}

\author{
S. Kračmar * \\ Š. Nečasová ${ }^{\dagger}$ \\ A. Novotný $\ddagger$
}

March 20, 2014

Department of Technical Mathematics, Czech Technical University,

Karlovo nám. 13, 12135 Prague 2, Czech Republic

Institute of Mathematics of the Academy of Sciences of the Czech Republic, Žitná 25, 11567 Praha 1, Czech Republic

IMATH, EA 2134, Université du Sud, Toulon Var, BP20132, 83957 La Garde, France

\begin{abstract}
We consider the motion of compressible viscous fluids around a rotating rigid obstacle when the velocity at infinity is non zero and parallel to the axis of rotation. We prove the existence of weak solution.
\end{abstract}

\section{Viscous compressible fluid in a rotating frame}

We consider a rigid body $\mathcal{S}$ with boundary $\Gamma$ such that $\Omega=\mathbb{R}^{3} \backslash \overline{\mathcal{S}}$ is an exterior domain. We assume that the body is rotating around the $x_{3}$-axis with the angular velocity $\omega=$ $(0,0,|\omega|)$. The position of the body at time $t>0$ is given by the formula

$$
\mathcal{S}(t)=\{y=O(t) x ; x \in \mathcal{S}\}, \quad \mathcal{S}(0)=\mathcal{S}
$$

${ }^{*}$ S. Kracmar acknowledges the support of the Czech Science Foundation (GAČR) project P201/11/1304

†S. Nečasová acknowledges the support of the Czech Science Foundation (GAČR) project P201/11/1304 in the general framework of RVO 67985840

$\ddagger$ The work was supported by the MODTERCOM project within the APEX programme of the region Provence-Alpes-Côte d'Azur, France 
where

$$
O(t)=\left(\begin{array}{ccc}
\cos |\omega| t & -\sin |\omega| t & 0 \\
\sin |\omega| t & \cos |\omega| t & 0 \\
0 & 0 & 1
\end{array}\right)
$$

We shall investigate the motion of viscous compressible fluid around the obstacle $\mathcal{S}(t)$. The exterior domain $\Omega(t)$ occupied by the viscous compressible fluid is time dependent:

$$
\begin{aligned}
& \Omega(t)=\{y=O(t) x ; x \in \Omega\} \\
& \Gamma(t)=\partial \Omega(t)=\{y=O(t) x ; x \in \Gamma\} .
\end{aligned}
$$

The fluid in $\Omega(t)$ is governed by the compressible Navier-Stokes equations. The unknowns, $\sigma, \mathbf{v}$ - density and velocity - solve the nonlinear system

$$
\begin{array}{rll}
\partial_{t}(\sigma \mathbf{v})+\operatorname{div}(\mathbf{v} \otimes \sigma \mathbf{v})+\nabla p(\sigma)-\operatorname{div} \mathbb{S}(\nabla \mathbf{v}) & =0 & \Omega(t), t \in(0, \infty) \\
\partial_{t} \sigma+\operatorname{div}(\sigma \mathbf{v}) & =0 & \text { in } \Omega(t), t \in(0, \infty) \\
\mathbf{v}(t, y) & =\omega \times y & \text { on } \Gamma(t), t \in(0, \infty) \\
\sigma(t, y) \rightarrow \varrho_{\infty}, \mathbf{v}(t, y) \rightarrow \mathbf{a}_{\infty} & \text { as } & |y| \rightarrow \infty
\end{array}
$$

completed with the initial conditions

$$
\sigma(0, y)=\sigma_{0}(y), \mathbf{v}(0, y)=\mathbf{v}_{0}(y), y \in \Omega .
$$

Here and hereafter, $\mathbb{S}$ is the viscous stress tensor - a linear function of $\nabla_{x} \mathbf{v}$ specified later, see (2.1), $p$ is the pressure -a given function of the density, see (2.2), $\mathbf{a}_{\infty} \in \mathbb{R}^{3}$ is a constant vector denoting a (prescribed) velocity of the flow at infinity and $\varrho_{\infty}>0$ is a (prescribed) positive constant density at infinity. We omit the action of external forces for the sake of simplicity.

We shall transform the time dependent domain $\Omega(t)$ to the fixed domain $\Omega$ by employing the change of variables

$$
x=O(t)^{T} y,
$$

and introducing new functions

$$
\mathbf{u}(t, x)=O(t)^{T} \mathbf{v}(t, y), \quad \rho(t, x)=\sigma(t, y) .
$$

It is easy to verify that

$$
O(-t)=O^{T}(t)=O^{-1}(t), O^{-1} \dot{O}=|\omega|\left(\begin{array}{rrr}
0 & -1 & 0 \\
1 & 0 & 0 \\
0 & 0 & 0
\end{array}\right)
$$




$$
O^{-1} \dot{O} x=\omega \times x, \dot{O}^{-1} O x=-\omega \times x .
$$

Moreover, the operations of inversion of the matrix $O(t)$ and of the derivative with respect to $t$ applied on $O(t)$ are commuting. Using these facts, we easily find that the first term of the left-hand side in $(1.4)_{1}$ reads in the new coordinates:

$$
\begin{aligned}
& \partial_{t}(\sigma \mathbf{v})(t, y)=\frac{\partial}{\partial t}\left((O(t) \rho \mathbf{u})\left(t, O^{-1}(t) y\right)\right) \\
= & \dot{O}(t) \rho \mathbf{u}\left(\left(t, O^{-1}(t) y\right)+O(t) \partial_{t}(\rho \mathbf{u})\left(t, O^{-1}(t) y\right)+O(t) \nabla_{x}(\rho \mathbf{u})\left(t, O^{-1}(t) y\right)\right) \dot{O}^{-1}(t) y \\
= & \left.O\left(O^{-1} \dot{O}\right) \rho \mathbf{u}(t, x)+O \partial_{t}(\rho \mathbf{u})(t, x)+O \nabla_{x}(\rho \mathbf{u})(t, x)\right) \dot{O}^{-1} O x \\
= & O\left[\partial_{t}(\rho \mathbf{u})-(\omega \times x) \cdot \nabla_{x}(\rho \mathbf{u})+\rho(\omega \times \mathbf{u})\right]=O\left[\partial_{t}(\rho \mathbf{u})-\operatorname{div}(\rho(\omega \times x) \otimes \mathbf{u})+\rho(\omega \times \mathbf{u})\right],
\end{aligned}
$$

where the following standard conventions have been used

$$
\begin{gathered}
\nabla_{x} \mathbf{u}=\left[a_{i j}\right]_{i, j=1,2,3}, a_{i j}=\frac{\partial u_{i}}{\partial x_{j}} \\
(\mathbf{z} \times A)_{k}=\sum_{j=1}^{3} z_{j} A_{j, k}, \mathbf{z}=\left[z_{1}, z_{2}, z_{3}\right], A=\left[A_{j k}\right]_{j, k=1,2,3} \\
\operatorname{div}_{\mathbf{x}}(\mathbf{z} \otimes \mathbf{w})=\nabla_{x} \cdot(\mathbf{z} \otimes \mathbf{w}), \mathbf{z} \otimes \mathbf{w}=\left[z_{i} w_{k}\right]_{i, k=1,2,3}
\end{gathered}
$$

Analogously,

$$
\operatorname{div}_{y}(\sigma \mathbf{v} \otimes \mathbf{v})=O \operatorname{div}_{x}(\rho \mathbf{u} \otimes \mathbf{u})
$$

and

$$
\operatorname{div}_{y} \mathbb{S}(\nabla \mathbf{v})(t, y)=O \operatorname{div}_{x} \mathbb{S}(\nabla \mathbf{u}) .
$$

Finally, we observe that the non homogenous Dirichlet boundary conditions $(1.4)_{3}$ transform to $\mathbf{u}=\omega \times x$, on the fixed boundary $\partial \mathcal{S}$ and the conditions at infinity remain unchanged. Consequently, after the change of variables (1.5) equations (1.4) transform to

$$
\begin{gathered}
\partial_{t}(\rho \mathbf{u})+\operatorname{div}(\rho(\mathbf{u}-\omega \times x) \otimes \mathbf{u})+\rho \omega \times \mathbf{u}+\nabla p(\rho)=\operatorname{div} \mathbb{S}(\nabla \mathbf{u}) \text { in }(0, T) \times \Omega \\
\partial_{t} \rho+\operatorname{div}(\rho(\mathbf{u}-\omega \times x))=0 \text { in }(0, T) \times \Omega \\
\mathbf{u}=\omega \times x \text { on }(0, T) \times \partial \Omega \\
\mathbf{u}(t, x) \rightarrow \mathbf{a}_{\infty} \in \mathbb{R}^{3}, \rho(t, x) \rightarrow \rho_{\infty}>0 \text { as }|x| \rightarrow \infty \\
\rho(0, x)=\rho_{0}(x),(\rho \mathbf{u})(0, x)=\mathbf{q}_{0}(x)=\varrho_{0} \mathbf{u}_{0}(x)
\end{gathered}
$$


This is a classical formulation of equations for unknown functions $(\rho, u)(t, x),(t, x) \in$ $(0, T) \times \Omega$ that is equivalent to the formulation (1.4). We shall deal with this problem in the following sections.

We refer for more details concerning the above change of variables to Galdi [14, Chapter 1], where the calculations are performed in the case of the (incompressible) Navier-Stokes equations.

Although the problem of the moving and rotating body in a compressible fluid is of obvious practical importance, to the best of our knowledge, there is no mathematical literature about the subject. Our goal in this paper is to investigate this problem with the large initial data and in the long time run, in the context of weak solutions in the spirit introduced to the theory of incompressible Navier-Stokes equations by Leray [22] and generalized later to compressible fluids in monographs by Lions [23], Feireisl [7] and in [11], [25]. Notice, that the case without rotation has been studied in [24], [25] and the case with zero velocity at infinity has been investigated in [26].

The "incompressible" analogue of this evolution problem is investigated in Hishida [19]. The reader can also consult Hieber and coauthors [18], and references quoted therein for the related results. The steady version of the incompressible case is investigated in Galdi and co-authors $[15,17,16]$, Farwig et al. see $[4,5,6]$. Variational approach was used in Deuring et al. [3] and in [21].

It is well known from the works of P.L. Lions [23], Feireisl [7] (see also [11], [25]), that the estimates available in the compressible models do not allow to pass to the limit in the non-linear terms in the equations. Instead, one has to use the methods of compensated compactness (div-curl lemma, effective viscous flux, various commutator lemmas, oscillations defect measure, renormalized solutions of the transport equation) and to search strong convergence within the structure of equations. We show, that after the transformation to a fixed domain one can adapt these techniques to the equations in the rotating frame. Although this choice is natural in the present context, there are many situations (e.g. domains with oscillating boundaries), where it is more convenient to work in the moving domain. We refer to $[12,13]$ and references quoted there, for relevant results.

Finally, notice that another situation, including Coriolis (and possibly a centrifugal force), when the fluid sticks or slips on the boundary of the body in the rotating frame and when the velocity at infinity tends to zero, enters into the framework of the existence results investigated in [20]. This context is typical of the models in the physics of atmosphere; it has been therefore broadly investigated in the context of singular limits, see e.g. Bresch, Chemin, Desjardins, Feireisl, Gallagher, Grenier, Gérard-Varet, Novotny and others, $[1,2,8,9,10]$.

The paper is organized as follows: In the first Section, we introduce the weak formulation and state the main result. The weak solutions are first established on large balls 
with help of invading domains via a penalization method described in Section 4 . In order to achieve the non zero prescribed velocity at infinity, the penalization uses an auxiliary vector field $\mathbf{u}_{\infty}$, that is constructed in Section 3.The limit process on large balls with the penalization parameter tending to infinity is performed in Section 5. Finally, the proof of the existence result is completed in Section 6, where the limit with the radius of large balls tending to infinity is established. The interesting feature in this limit process appears in the renormalized continuity equation, where the test functions have to be chosen in such a way in order to eliminate the possible contributions of the terms containing $\omega \times x$, (that explode at infinity) to the oscillations in the density sequence. This issue is handled in the last part of Section 6 .

\section{Setting of the problem, weak solutions}

Before introducing the notion of weak solutions of the problem (1.7-1.10) and in order to determine the appropriate functional spaces, we shall specify the form of the viscous stress tensor and the pressure. Following the standard existence theory in the situation without rotation, we deal with the Newtonian fluids, where

$$
\mathbb{S}\left(\nabla_{x} \mathbf{u}\right)=\mu \mathbb{T}\left(\nabla_{x} \mathbf{u}\right)+\eta \operatorname{div}_{x} \mathbf{u} \mathbb{I}, \quad \mathbb{T}\left(\nabla_{x} \mathbf{u}\right)=\nabla_{x} \mathbf{u}+\nabla_{x}^{T} \mathbf{u}-\frac{2}{3} \operatorname{div}_{x} \mathbf{u} \mathbb{I},
$$

with $\mu>0, \eta \geq 0$. Moreover, we assume that the pressure is a given function of the density that satisfies hypotheses

$$
p \in C[0, \infty) \cap C^{1}(0, \infty), p(0)=0, p^{\prime}(\varrho)>0 \text { for all } \varrho>0, \lim _{\varrho \rightarrow \infty} \frac{p^{\prime}(\varrho)}{\varrho^{\gamma-1}}=a>0
$$

for a certain $\gamma>3 / 2$. Notice that this assumption covers at least one physically reasonable case, namely isentropic flows of a monoatomic gas.

In view of these assumptions, the weak formulation of problem (1.7-1.10) reads:

\section{Definition 1}

We shall call a couple $(\rho, \mathbf{u})$ a renormalized weak solution of problem (1.7-2.2) if:

(i) Functions $(\varrho, \mathbf{u})$ are such that

$$
\begin{gathered}
\rho \geq 0, \quad \rho-\varrho_{\infty} \in L^{\infty}\left(0, T ; L^{\gamma}+L^{2}(\Omega)\right), \\
\varrho\left(\mathbf{u}-\mathbf{a}_{\infty}\right)^{2} \in L^{\infty}\left(0, T ; L^{1}(\Omega)\right), \\
\mathbf{u}-\mathbf{a}_{\infty} \in L^{2}\left(0, T ; W^{1,2}(\Omega)\right),\left.\quad \mathbf{u}(t)\right|_{\partial \Omega}=\omega \times x \text { for a.a. } t \in(0, T) .
\end{gathered}
$$


(ii) Density $\varrho \in C_{\text {weak }}\left([0, T] ; L^{\gamma}(K)\right)$ for any compact $K \subset \bar{\Omega}$ and the equation of continuity (1.8) is satisfied in the weak sense,

$$
\left.\int_{\Omega} \varrho \varphi \mathrm{d} x\right|_{0} ^{\tau}=\int_{0}^{\tau} \int_{\Omega} \varrho \partial_{t} \varphi+\varrho(\mathbf{u}-\omega \times x) \cdot \nabla_{x} \varphi \mathrm{d} x \mathrm{~d} t
$$

for all $\tau \in[0, T]$ and any test function $\varphi \in C_{c}^{\infty}([0, T] \times \bar{\Omega})$, as well as in the renormalized weak sense

$$
\begin{aligned}
\left.\int_{\Omega} b(\varrho) \varphi \mathrm{d} x\right|_{0} ^{\tau} & =\int_{0}^{\tau} \int_{\Omega} b(\varrho)\left(\partial_{t} \varphi+(\mathbf{u}-\omega \times x) \cdot \nabla_{x} \varphi\right) \mathrm{d} x \mathrm{~d} t \\
& +\int_{0}^{\tau} \int_{\Omega}\left(b(\varrho)-\varrho b^{\prime}(\varrho)\right) \operatorname{div} \mathbf{u} \varphi \mathrm{d} x \mathrm{~d} t
\end{aligned}
$$

for any $\tau \in[0, T]$, for any $\varphi \in C_{c}^{1}([0, T] \times \bar{\Omega})$ and for all

$$
b \in C_{c}[0, \infty) \cap C_{c}^{1}(0, \infty)
$$

Here and in what follows $\left.\int_{\Omega} g \varphi \mathrm{d} x\right|_{0} ^{\tau}=\int_{\Omega} g(\tau, x) \varphi(\tau, x) \mathrm{d} x-\int_{\Omega} g_{0}(x) \varphi(0, x) \mathrm{d} x$.

(iii) The linear momentum $\varrho \mathbf{u} \in C_{\text {weak }}\left([0, T], L^{2 \gamma /(\gamma+1)}(K)\right)$ for any compact $K \subset \bar{\Omega}$ and the momentum equation (1.7) is satisfied in the weak sense

$$
\begin{gathered}
\left.\int_{\Omega} \varrho \mathbf{u} \cdot \varphi \mathrm{d} x\right|_{0} ^{\tau}+\int_{0}^{\tau} \int_{\Omega} \mathbb{S}\left(\nabla_{x} \mathbf{u}\right): \nabla_{x} \varphi \mathrm{d} x \mathrm{~d} t \\
=\int_{0}^{\tau} \int_{\Omega}\left(\varrho \mathbf{u} \cdot \partial_{t} \varphi+\varrho(\mathbf{u}-\omega \times x) \otimes \mathbf{u}: \nabla_{x} \varphi-\varrho(\omega \times \mathbf{u}) \cdot \varphi+p(\varrho) \operatorname{div}_{x} \varphi\right) \mathrm{d} x \mathrm{~d} t
\end{gathered}
$$

for all $\tau \in[0, T]$ and for any test function $\varphi \in C_{c}^{\infty}([0, T] \times \Omega)$.

Notice that the class (2.6) of the renormalizing functions $b$ can be enlarged to

$$
b \in C[0, \infty) \cap C^{1}(0, \infty), z b^{\prime}(z) \in L^{\infty}(0,1), b(z) / z^{5 \gamma / 6},\left(z b^{\prime}-b\right)(z) / z^{\gamma / 2} \in L^{\infty}(1, \infty)
$$

by using the Lebesgue dominated convergence theorem.

Here and hereafter, we denote

$$
\begin{gathered}
H(\rho)=\rho \int_{1}^{\rho} \frac{p(s)}{s^{2}} d s \\
E\left(\varrho \mid \varrho_{\infty}\right)=H(\varrho)-H^{\prime}\left(\varrho_{\infty}\right)\left(\varrho-\varrho_{\infty}\right)-H\left(\varrho_{\infty}\right) .
\end{gathered}
$$


We are now in a position to state the main existence result.

Theorem (Existence of weak solutions) Assume that $\rho_{\infty}>0$ and $\Omega=\mathbb{R}^{3} \backslash \overline{\mathcal{S}}$, where $\mathcal{S}$ is a bounded Lipschitz domain. Suppose that the vector $\omega$ is parallel to the vector $\mathbf{a}_{\infty}$, and that the viscous stress tensor $\mathbb{S}$ and the pressure $p$ satisfy (2.1), (2.2). Assume that

$$
\varrho_{0} \geq 0, \quad E\left(\rho_{0} \mid \varrho_{\infty}\right) \in L^{1}(\Omega) \quad \varrho_{0}\left(\mathbf{u}_{0}-\mathbf{a}_{\infty}\right)^{2} \in L^{1}(\Omega) .
$$

Then the problem (1.7-1.11) admits at least one renormalized weak solution.

\section{An auxiliary result}

Let us denote the open ball with radius $r$ centered at the origin by $B_{r}$. Suppose without loss of generality that $\overline{\mathcal{S}} \subset B_{1 / 2}$ and $B_{1} \subset B_{R}$. To begin, we claim that there exists a smooth vector field $\mathbf{u}_{\infty} \in C_{c}^{\infty}\left(\mathbb{R}^{3}\right)$ such that:

$$
\begin{aligned}
& \mathbf{u}_{\infty}=\left\{\begin{array}{l}
\omega \times x \text { in } \mathcal{S} \\
\operatorname{supp} \mathbf{u}_{\infty} \subset B_{2 R} \\
\mathbf{a}_{\infty} \text { in } B_{(3 / 2) R} \backslash B_{1}
\end{array}\right. \\
& \operatorname{div} \mathbf{u}_{\infty}=0 \text { in } \mathcal{S} \cup\left(\mathbb{R}^{3} \backslash B_{1}\right)
\end{aligned}
$$

Let us consider non-increasing functions $\Phi_{1}, \Phi_{2}, \Psi_{R} \in C_{c}^{\infty}([0,+\infty))$ as follows: $0 \leq \Phi_{1} \leq$ $1, \Phi_{1}=1$ in $[0,5 / 9), \Phi_{1}=0$ in $[2 / 3,+\infty), 0 \leq \Phi_{2} \leq 1, \Phi_{2}=1$ in $[0,7 / 9), \Phi_{2}=0$ in $[8 / 9,+\infty), 0 \leq \Psi_{R} \leq 1, \Psi_{R}=1$ in $\left.[0,(3 / 2) R)\right), \Psi_{R}=0$ in $[2 R-1 / 4,+\infty)$. Let us set

$$
\tilde{\mathbf{a}}_{\infty}(x)=\mathbf{a}_{\infty}\left(1-\Phi_{2}(|x|)\right) \Psi_{R}(|x|) .
$$

Using the classical Stokes formula it is easy to check that

$$
\int_{B_{2 R-1 / 4} \backslash B_{(3 / 2) R}} \operatorname{div} \tilde{\mathbf{a}}_{\infty}(x) \mathrm{d} x=0 .
$$

So, from Lemma 3.1 (see further) we get that there exists a vector field $\mathbf{b}_{\infty} \in$ $W_{0}^{1, p}\left(B_{2 R-1 / 4} \backslash B_{(3 / 2) R}\right), \quad p \in(1,+\infty)$ and

$$
\operatorname{div} \mathbf{b}_{\infty}(x)=\operatorname{div} \tilde{\mathbf{a}}_{\infty}(x) \text { for } x \in B_{2 R-1 / 4} \backslash B_{(3 / 2) R} .
$$

It is easy to see that divergence of the expression $(\omega \times x) \Phi_{1}(|x|) \in C_{c}^{\infty}\left(\mathbb{R}^{3}\right)$ is zero. Now, let extend $\mathbf{b}_{\infty}(\cdot)$ by zero in $\mathbb{R}^{3}$ and set

$$
\mathbf{u}_{\infty}(x)=(\omega \times x) \Phi_{1}(|x|)+m_{\varepsilon} *\left(\tilde{\mathbf{a}}_{\infty}-\mathbf{b}_{\infty}\right)(x),
$$


where $0<\varepsilon<1 / 8$ is fixed and $m_{\varepsilon}(\cdot)$ is the standard mollifier. In the previous reasoning, we have used the auxiliary result concerning the so called Bogovskiĩ operator (see e.g. Galdi [15]):

Lemma 3.1. Let $G \subset \mathbb{R}^{N}$ be a bounded domain with Lipschitz boundary. Then there exists a linear operator $\mathcal{B}_{G}=\left(\mathcal{B}_{G}^{(1)}, \mathcal{B}_{G}^{(2)}, \ldots \mathcal{B}_{G}^{(N)}\right)$ with the following properties:

1.

$$
\mathcal{B}_{G}:\left\{\mathcal{F} \in L^{q}(G) ; \quad \int_{G} \mathcal{F} d x=0\right\} \longrightarrow W_{0}^{1, q}\left(G ; \mathbb{R}^{N}\right)
$$

is a bounded linear operator. More precisely, for any $q \in(1, \infty)$,

$$
\left\|\nabla \mathcal{B}_{G}(\mathcal{F})\right\|_{L^{q}\left(G ; \mathbb{R}^{N \times N}\right)} \leq c(G, q)\|\mathcal{F}\|_{L^{q}(G)}
$$

2. the function $\mathbf{v}=\mathcal{B}_{G}(\mathcal{F})$ solves the problem

$$
\operatorname{div} \mathbf{v}=\mathcal{F} \text { in } G,\left.\quad \mathbf{v}\right|_{\partial G}=0
$$

3. if, moreover, $\mathcal{F}$ can be written in the form $\mathcal{F}=\operatorname{div} \mathbf{Q}$ for a certain $\mathbf{Q} \in L^{p}\left(G, \mathbb{R}^{N}\right)$ satisfying $\left.\mathbf{Q} \cdot \mathbf{n}\right|_{\partial G}=0$ where $\mathbf{n}$ stands for the outward unit normal vector to $\partial G$, then it holds

$$
\left\|\mathcal{B}_{G}(\mathcal{F})\right\|_{L^{p}\left(G ; \mathbb{R}^{N}\right)} \leq c(G, p)\|\mathbf{Q}\|_{L^{p}(G)}
$$

\section{Penalized problem}

In what follows, we denote

$$
V=B_{2 R}
$$

and extend $\varrho_{0}$ by 0 and $\mathbf{u}_{0}$ by $\omega \times x$ in $\mathcal{S}$. We shall solve on $(0, T) \times V$ the following penalized problem

$$
\begin{gathered}
\partial_{t}(\rho \mathbf{u})+\operatorname{div}((\rho(\mathbf{u}-\omega \times x) \otimes \mathbf{u}))+\rho \omega \times \mathbf{u}-\operatorname{div} \mathbb{S}(\nabla \mathbf{u}) \\
+\nabla p(\rho)+k \cdot 1_{\left\{\left(V \backslash B_{R}\right) \cup \mathcal{S}\right\}}\left(\mathbf{u}-\mathbf{u}_{\infty}\right)=0 \\
\partial_{t} \rho+\operatorname{div}(\rho(\mathbf{u}-\omega \times x))=0 . \\
\left.\mathbf{u}\right|_{\partial V}=0 \\
\varrho(0)=\varrho_{0}, \quad \varrho \mathbf{u}(0)=\varrho_{0} \mathbf{u}_{0} .
\end{gathered}
$$


Definition 4.1. A couple $(\varrho, \mathbf{u})$ is a renormalized finite energy weak solution of the penalized problem (4.1-4.4) if

(i)

$$
\begin{gathered}
\rho \geq 0, \rho-\varrho_{\infty} \in L^{\infty}\left(0, T ;\left[L^{\gamma}+L^{2}\right](V)\right), \\
\varrho\left(\mathbf{u}-\mathbf{u}_{\infty}\right)^{2} \in L^{\infty}\left(0, T ; L^{1}(V)\right), \\
\mathbf{u}-\mathbf{u}_{\infty} \in L^{2}\left(0, T ; W_{0}^{1,2}\left(V ; \mathbb{R}^{3}\right)\right)
\end{gathered}
$$

(ii) Equation of continuity (1.8) is satisfied in the weak sense, see (2.4) with $\Omega$ replaced by $V$ for any test function $\varphi \in C_{c}^{\infty}([0, T] \times \bar{V})$, as well as in the renormalized weak sense, see (2.5) with $\Omega$ replaced by $V$ for any $b$ as in (2.5) and any $\varphi \in C_{c}^{1}([0, T) \times \bar{V})$.

(iii) Momentum equation is satisfied in the weak sense,

$$
\begin{gathered}
\int_{0}^{\tau} \int_{\Omega}\left(\varrho \mathbf{u} \cdot \partial_{t} \varphi+\varrho(\mathbf{u}-\omega \times x) \otimes \mathbf{u}: \nabla_{x} \varphi-\varrho(\omega \times \mathbf{u}) \cdot \varphi-k \cdot 1_{\left\{\left(V \backslash B_{R}\right) \cup S\right\}}\left(\mathbf{u}-\mathbf{u}_{\infty}\right) \cdot \varphi\right. \\
\left.+p(\varrho) \operatorname{div}_{x} \varphi\right) \mathrm{d} x \mathrm{~d} t=\left.\int_{\Omega} \varrho \mathbf{u} \varphi \mathrm{d} x\right|_{0} ^{\tau}+\int_{0}^{\tau} \int_{\Omega} \mathbb{S}\left(\nabla_{x} \mathbf{u}\right): \nabla_{x} \varphi \mathrm{d} x \mathrm{~d} t
\end{gathered}
$$

for all $\tau \in[0, T]$ and for any test function $\varphi \in C_{c}^{\infty}([0, T] \times V)$.

(iv) The following energy inequality is satisfied

$$
\begin{gathered}
\left.\int_{V}\left(\frac{1}{2} \varrho\left|\mathbf{u}-\mathbf{u}_{\infty}\right|^{2}+E\left(\varrho \mid \varrho_{\infty}\right)\right) \mathrm{d} x\right|_{0} ^{\tau} \\
+\int_{0}^{\tau} \int_{V}\left(\mathbb{S}\left(\nabla_{x}\left(\mathbf{u}-\mathbf{u}_{\infty}\right)\right): \nabla_{x}\left(\mathbf{u}-\mathbf{u}_{\infty}\right)+k 1_{\left(V \backslash B_{R}\right) \cup S}\left|\mathbf{u}-\mathbf{u}_{\infty}\right|^{2}\right) \mathrm{d} x \mathrm{~d} t \leq \\
-\int_{0}^{\tau} \int_{V} p(\rho) \operatorname{div} \mathbf{u}_{\infty}+\int_{0}^{\tau} \int_{V} \rho(\mathbf{u}-\omega \times x) \cdot \nabla_{x} \mathbf{u}_{\infty} \cdot\left(\mathbf{u}_{\infty}-\mathbf{u}\right) \mathrm{d} x \mathrm{~d} t \\
-\int_{0}^{\tau} \int_{V} \varrho\left(\omega \times \mathbf{u}_{\infty}\right) \cdot\left(\mathbf{u}-\mathbf{u}_{\infty}\right) \mathrm{d} x \mathrm{~d} t-\int_{0}^{\tau} \int_{V} \mathbb{S}\left(\nabla_{x} \mathbf{u}_{\infty}\right): \nabla_{x}\left(\mathbf{u}-\mathbf{u}_{\infty}\right) \mathrm{d} x \mathrm{~d} t
\end{gathered}
$$

for a. a. $\tau \in(0, T)$.

Under assumptions (2.11) with $\mathbf{u}_{\infty}$ defined in formulas (3.1-3.2), problem (4.1-4.2) admits at least one weak solution $\left(\varrho_{k}, \mathbf{u}_{k}\right)$. This result can be proved similarly as in Theorems 7.7, 7.79 [25, Sections 7.1.2, 7.12.6]. 


\section{$5 \quad$ Limit $k \rightarrow \infty$}

We deduce from estimate (4.7) the following estimates, with $c=c(R)$ a generic constant that may depend on $R$ but is independent of $k$ :

$$
\begin{gathered}
\operatorname{ess} \sup _{t \in(0, T)} \int_{V} \rho_{k}\left|\mathbf{u}_{k}-\mathbf{u}_{\infty}\right|^{2} \mathrm{~d} x \leq c(R) \\
\operatorname{ess} \sup _{t \in(0, T)} \int_{V} E\left(\rho_{k} \mid \rho_{\infty}\right) \mathrm{d} x \leq c(R) \\
\left\|\mathbf{u}_{k}\right\|_{L^{2}\left(0, T ; W^{1,2}\left(V ; \mathbb{R}^{3}\right)\right)} \leq c(R) \\
\left\|\mathbf{u}_{k}-\mathbf{u}_{\infty}\right\|_{L^{2}\left((0, T) \times\left(\left(V \backslash B_{R}\right) \cup \mathcal{S}\right) ; \mathbb{R}^{3}\right)} \leq \frac{c(R)}{\sqrt{k}}
\end{gathered}
$$

When deducing (5.3) we have used the Korn-Poincaré type inequality in the form

$$
\left\|\mathbb{S}\left(\nabla\left(\mathbf{u}_{k}-\mathbf{u}_{\infty}\right)\right)\right\|_{L^{2}\left(V ; \mathbb{R}^{3 \times 3}\right)} \geq c\left\|\mathbf{u}_{k}-\mathbf{u}_{\infty}\right\|_{W_{0}^{1,2}\left(V ; \mathbb{R}^{3 \times 3}\right)},
$$

see Theorem 10.16 in [11]. Finally, introducing for any $h \in L^{1}((0, T) \times V)$

$$
[h]_{\text {ess }}=1_{\left\{\left|\rho-\rho_{\infty}\right| \leq \rho_{\infty} / 2\right\}} \cdot h, \quad[h]_{\text {res }}=h-h_{\text {ess }}
$$

and taking into account

$$
E\left(\rho \mid \rho_{\infty}\right) \geq c\left(\left|\rho-\rho_{\infty}\right|^{2} \cdot 1_{\left\{\rho_{\infty} / 2 \leq \rho \leq 2 \rho_{\infty}\right\}}+\left(1+\rho^{\gamma}\right) \cdot 1_{\left\{\rho<\rho_{\infty} / 2 \text { or } \rho>2 \rho_{\infty}\right\}}\right)
$$

(see e.g. [11]) we get from (5.2):

$$
\begin{gathered}
\operatorname{ess} \sup _{t \in(0, T)}\left\|\left[\rho_{k}-\rho_{\infty}\right]_{e s s}\right\|_{L^{2}(V)} \leq c(R), \\
\text { ess sup } \operatorname{sic}_{t \in(0, T)}\left\|\left[\rho_{k}\right]_{\text {res }}\right\|_{L^{\gamma}(V)} \leq c(R) .
\end{gathered}
$$

On the basis of these estimates we get the existence of a subsequence $\left(\varrho_{k}, \mathbf{u}_{k}\right)$ and a couple $(\varrho, \mathbf{u})$ such that

$$
\begin{gathered}
\varrho_{k}-\varrho_{\infty} \rightarrow \varrho-\varrho_{\infty} \text { in } L^{\infty}\left(0, T ;\left[L^{2}+L^{\gamma}\right](V)\right) \\
\mathbf{u}_{k} \rightarrow \mathbf{u} \text { in } L^{2}\left(0, T ; W^{1,2}\left(V, \mathbb{R}^{3}\right)\right),
\end{gathered}
$$

where $\varrho \geq 0$. It can be proved via quite recent but nowadays classical methods (including effective viscous flux, oscillations defect measures, renormalized solutions of continuity equation) [23], [7], [25], [11] that the couple $(\varrho, \mathbf{u})$ has the following properties ${ }^{1}$ :

\footnotetext{
${ }^{1}$ We shall give more details on this procedure when treating the limit $R \rightarrow \infty$ in the next section
} 
(i) $\varrho \in C_{\text {weak }}\left(0, T ; L^{\gamma}(V)\right)$ and the continuity equation is satisfied in the weak sense

$$
\left.\int_{V} \varrho \varphi \mathrm{d} x\right|_{0} ^{\tau}=\int_{0}^{\tau} \int_{V} \varrho \partial_{t} \varphi+\varrho(\mathbf{u}-\omega \times x) \cdot \nabla_{x} \varphi \mathrm{d} x \mathrm{~d} t
$$

for all $\tau \in[0, T]$ and any $\varphi \in C_{c}^{\infty}([0, T] \times V)$. Similarly, the renormalized continuity equation is satisfied in the form:

$$
\begin{gathered}
\left.\int_{V} b(\varrho) \varphi \mathrm{d} x\right|_{0} ^{\tau}+\int_{0}^{\tau} \int_{V}\left(\varrho b^{\prime}(\varrho)-b(\varrho)\right) \operatorname{div} \mathbf{u} \varphi \mathrm{d} x \mathrm{~d} t= \\
\int_{0}^{\tau} \int_{V} b(\varrho)\left(\partial_{t} \varphi+(\mathbf{u}-\omega \times x) \cdot \nabla_{x} \varphi\right) \mathrm{d} x \mathrm{~d} t
\end{gathered}
$$

for all $\tau \in[0, T]$, for any $b$ belonging to the class (2.6) and for any

$$
\varphi \in C_{c}^{1}([0, T] \times V) .
$$

(ii) Since $\mathbf{u}-\omega \times x=0$ a.e. in $(0, T) \times S$, equation (5.8) yields, in particular,

$$
\int_{0}^{T} \eta^{\prime}(t) \int_{S} \varrho \varphi(x) \mathrm{d} x \mathrm{~d} t=0
$$

for all $\eta \in C^{1}(0, T)$ and for all $\varphi \in C_{c}^{1}(\mathcal{S})$. This means that

$$
\text { for a.a. }(t, x) \in(0, T) \times \mathcal{S}, \quad \varrho(t, x)=\varrho_{0}(x)=0 .
$$

With this information at hand, formula (5.8) yields, in particular,

$$
\left.\int_{\Omega_{R}} \varrho \varphi \mathrm{d} x\right|_{0} ^{\tau}=\int_{0}^{\tau} \int_{\Omega_{R}} \varrho\left(\partial_{t} \varphi+(\mathbf{u}-\omega \times x) \cdot \nabla_{x} \varphi\right) \mathrm{d} x \mathrm{~d} t
$$

for all $\tau \in[0, T]$ and for any test function $\varphi \in C_{c}^{\infty}\left([0, T] \times\left(B_{R} \backslash S\right)\right)$. Here and in what follows, we denote $\Omega_{R}=\Omega \cap B_{R}=B_{R} \backslash \overline{\mathcal{S}}$. Similarly, formula (5.9) yields

$$
\begin{gathered}
\left.\int_{\Omega_{R}} b(\varrho) \varphi \mathrm{d} x\right|_{0} ^{\tau}=\int_{0}^{\tau} \int_{\Omega_{R}}\left(b(\varrho)-\varrho b^{\prime}(\varrho)\right) \operatorname{div} \mathbf{u} \varphi \mathrm{d} x \mathrm{~d} t \\
\int_{0}^{\tau} \int_{\Omega_{R}} b(\varrho)\left(\partial_{t} \varphi+(\mathbf{u}-\omega \times x) \cdot \nabla_{x} \varphi\right) \mathrm{d} x \mathrm{~d} t
\end{gathered}
$$

for all $\tau \in[0, T]$, for any $b$ belonging to the class (2.6) and

$$
\varphi \in C_{c}^{1}\left([0, T] \times\left(B_{R} \backslash \mathcal{S}\right)\right) .
$$


(iii) There holds $\varrho \mathbf{u} \in C_{\text {weak }}\left(0, T ; L^{2 \gamma / \gamma+1}\left(V ; \mathbb{R}^{3}\right)\right)$ and

$$
\begin{aligned}
\int_{0}^{T} \int_{\Omega_{R}}\left(\varrho \mathbf{u} \cdot \partial_{t} \varphi\right. & \left.+\varrho(\mathbf{u}-\omega \times x) \otimes \mathbf{u}: \nabla_{x} \varphi-\varrho(\omega \times \mathbf{u}) \cdot \varphi+p(\varrho) \operatorname{div}{ }_{x} \varphi\right) \mathrm{d} x \mathrm{~d} t \\
& =\left.\int_{\Omega_{R}} \varrho \mathbf{u} \varphi \mathrm{d} x\right|_{0} ^{\tau}+\int_{0}^{T} \int_{\Omega_{R}} \mathbb{S}\left(\nabla_{x} \mathbf{u}\right): \nabla_{x} \varphi \mathrm{d} x \mathrm{~d} t
\end{aligned}
$$

for any $\tau \in[0, T]$ and all test functions $\varphi \in C_{c}^{\infty}\left([0, T] \times \Omega_{R}\right)$. Moreover,

$$
\mathbf{u}=\mathbf{u}_{\infty} \text { a.e. in }(0, T) \times\left[\left(V \backslash B_{R}\right) \cup \mathcal{S}\right] .
$$

(iv) The following energy inequality is satisfied

$$
\begin{gathered}
\qquad\left.\int_{\Omega_{R}}\left(\frac{1}{2} \varrho\left|\mathbf{u}-\mathbf{u}_{\infty}\right|^{2}+E\left(\varrho \mid \varrho_{\infty}\right)\right) \mathrm{d} x\right|_{0} ^{\tau} \\
+\int_{0}^{\tau} \int_{\Omega_{R}}\left(\mathbb{S}\left(\nabla_{x}\left(\mathbf{u}-\mathbf{u}_{\infty}\right)\right): \nabla_{x}\left(\mathbf{u}-\mathbf{u}_{\infty}\right) \mathrm{d} x \mathrm{~d} t \leq\right. \\
-\int_{0}^{\tau} \int_{B_{1} \backslash S} p(\rho) \operatorname{div} \mathbf{u}_{\infty}-\int_{0}^{\tau} \int_{B_{1} \backslash S} \rho(\mathbf{u}-\omega \times x) \cdot \nabla_{x} \mathbf{u}_{\infty} \cdot\left(\mathbf{u}-\mathbf{u}_{\infty}\right) \mathrm{d} x \mathrm{~d} t \\
-\int_{0}^{\tau} \int_{B_{1} \backslash S} \varrho\left(\omega \times \mathbf{u}_{\infty}\right) \cdot\left(\mathbf{u}-\mathbf{u}_{\infty}\right) \mathrm{d} x \mathrm{~d} t-\int_{0}^{\tau} \int_{B_{1} \backslash S} \mathbb{S}\left(\nabla_{x} \mathbf{u}_{\infty}\right): \nabla_{x}\left(\mathbf{u}-\mathbf{u}_{\infty}\right) \mathrm{d} x \mathrm{~d} t
\end{gathered}
$$

When deriving (5.14) we have used the facts that: 1) $\omega$ and $\mathbf{u}_{\infty}$ are parallel if $x \in$ $B_{\frac{3}{2} R} \backslash B_{1}$ and 2) properties (3.1), (3.2) and (5.13).

\section{$6 \quad$ Limit $R \rightarrow \infty$}

\subsection{Weak limits}

We set

$$
\mathbf{U}_{\infty}=\left\{\begin{array}{c}
\mathbf{u}_{\infty} \text { in } B_{1} \\
\mathbf{a}_{\infty} \text { in } R^{3} \backslash B_{1}
\end{array}\right\} .
$$

Here and hereafter we extend $\rho$ by $\rho_{\infty}$ outside $B_{R}$ (and denote the new function by $\varrho$ ), and $\mathbf{u}$ by $\mathbf{U}_{\infty}$ outside $B_{R}$ and denote the new function again by $\mathbf{u}$. Using (5.1-5.7) we can deduce from (5.14) estimates: 


$$
\begin{gathered}
\int_{\mathbb{R}^{3}}[1]_{\mathrm{res}} \mathrm{d} x \leq c \\
\operatorname{ess} \sup _{t \in(0, T)}\left\|\left[\rho_{R}-\rho_{\infty}\right]_{e s s}\right\|_{L^{2}\left(\mathbb{R}^{3}\right)} \leq c \\
\operatorname{ess} \sup _{t \in(0, T)}\left\|\left[\rho_{R}\right]_{\text {res }}\right\|_{L^{\gamma}\left(\mathbb{R}^{3}\right)} \leq c \\
\left.\operatorname{ess} \sup _{t \in(0, T)}\left\|\varrho_{R}\left|\mathbf{u}_{R}-\mathbf{U}_{\infty}\right|^{2}\right\|_{L^{1}\left(R^{3}\right.}\right)=0 . \\
\left\|\mathbf{u}_{R}-\mathbf{U}_{\infty}\right\|_{L^{2}\left((0, T) \times W^{1,2}\left(\mathbb{R}^{3} ; R^{3}\right)\right)} \leq c
\end{gathered}
$$

Hereafter, we describe in a concise form the nowadays classical argument including effective viscous flux identity, oscillations defect measures and renormalized continuity equation leading to the proof of the main theorem. It is to be noticed that the main issue is to show the strong convergence of the density sequence.

We use in momentum equation (5.12) (written with $\left(\varrho_{R}, \vartheta_{R}, \mathbf{u}_{R}\right)$ on $\left.\Omega_{R}\right)$ with the test function $\varphi=\mathcal{B}_{\Omega_{n}}\left(\varrho_{R}^{\nu}\right) \in L^{\infty}\left(0, T ; W_{0}^{1, \gamma / \nu}\left(\Omega_{n} ; R^{3}\right)\right) \cap L^{2}\left(0, T ; L^{p}\left(\Omega_{n}\right)\right), \frac{\nu}{\gamma}=\frac{1}{2}=\frac{1}{p}$, where $\nu>0$ is sufficiently small and $n \in \mathbb{N}$ is fixed. After some technical calculations similar to [11, Section 2.2.5, Appendix], where one uses the Bogovskii lemma 3.1, one derives that there exists $\nu>0$ such that for all $R \geq n$,

$$
\int_{0}^{T} \int_{\Omega_{n}} \varrho_{R}^{\gamma+\nu} \mathrm{d} x \mathrm{~d} t \leq c(n)
$$

Proceeding as in [25, Section 7.12.6] we deduce from estimates (6.2-6.7), by means of a diagonalisation procedure, existence of a couple $(\varrho, \mathbf{u})$ such that

$$
\begin{gathered}
\varrho_{R}-\varrho_{\infty} \rightarrow_{*} \varrho-\varrho_{\infty} \text { in } L^{\infty}\left(0, T ; L^{\gamma}+L^{2}(\Omega)\right), \\
\mathbf{u}_{R}-\mathbf{U}_{\infty} \rightarrow \mathbf{u}-\mathbf{U}_{\infty} \text { in } L^{2}\left(0, T ; W^{1,2}\left(\Omega ; \mathbb{R}^{3}\right)\right), \\
p\left(\varrho_{R}\right) \rightarrow \overline{p(\varrho)} \text { in } L^{q}\left((0, T) \times \Omega_{n}\right) \text { with some } q>1, \forall n \in N,
\end{gathered}
$$

as $R \rightarrow \infty$. Here and hereafter $\overline{g(\varrho, \mathbf{u})}$ denotes $L^{1}$ weak limit of the sequence $g\left(\varrho_{R}, \mathbf{u}_{R}\right)$.

Moreover, using continuity equation (5.10), renormalized continuity equation (5.9) and momentum equation (5.12) together with estimate (6.3-6.5) (all written with $\left(\varrho_{R}, \mathbf{u}_{R}\right)$ on 
$\left.\Omega_{R}\right)$ ), we get, after employing the Arzela-Ascoli compactness argument and a density argument,

$$
\begin{gathered}
\varrho_{R} \rightarrow \varrho \text { in } C_{\text {weak }}\left([0, T] ; L^{\gamma}\left(\Omega_{n}\right)\right) \text { and in } L^{2}\left(0, T ; W^{-1,2}\left(\Omega_{n}\right)\right), \forall n \in N, \\
h\left(\varrho_{R}\right) \rightarrow \overline{h(\varrho)} \text { in } C_{\text {weak }}\left([0, T] ; L^{q}\left(\Omega_{n}\right)\right), q \in[1, \infty) \text { and in } L^{2}\left(0, T ; W^{-1,2}\left(\Omega_{n}\right)\right), \forall n \in N,
\end{gathered}
$$

where $h$ belongs to $(2.6)$,

$$
\begin{gathered}
\varrho_{R} \mathbf{u}_{R} \rightarrow \varrho \mathbf{u} \text { in } C_{\text {weak }}\left([0, T] ; L^{2 \gamma /(\gamma+1)}\left(\Omega_{n} ; \mathbb{R}^{3}\right)\right) \text { and in } L^{2}\left(0, T ; W^{-1,2}\left(\Omega_{n}, \mathbb{R}^{3}\right)\right), \forall n \in N, \\
\varrho_{R} \mathbf{u}_{R} \otimes \mathbf{u}_{R} \rightarrow \varrho \mathbf{u} \otimes \mathbf{u} \text { in } L^{2}\left(0, T ; L^{6 \gamma /(4 \gamma+3)}\left(\Omega_{n}, \mathbb{R}^{3 \times 3}\right)\right), \forall n \in N
\end{gathered}
$$

In all three cases, the strong $L^{2} W^{-1,2}$ convergence follows from the previous $C_{\text {weak }} L^{q}$ convergence and the compact embedding $L^{q}(G) \hookrightarrow \hookrightarrow W^{-1,2}(G), q>6 / 5$, where $G$ is a bounded domain.

At this stage, we may pass to the limit $R \rightarrow \infty$ in equations (5.12), (5.10) and (5.9) that are written with $\left(\varrho_{R}, \vartheta_{R}, \mathbf{u}_{R}\right)$ on $\Omega_{R}$. We get

$$
\left.\int_{\Omega} \varrho \varphi \mathrm{d} x\right|_{0} ^{\tau}=\int_{0}^{\tau} \int_{\Omega}\left(\varrho \partial_{t} \varphi+\varrho(\mathbf{u}-\omega \times x) \cdot \nabla_{x} \varphi\right) \mathrm{d} x \mathrm{~d} t
$$

for all $\tau \in[0, T]$ and for any $\varphi \in C_{c}^{1}([0, T] \times \bar{\Omega})$;

$$
\begin{gathered}
\left.\int_{\Omega} \varrho \mathbf{u} \cdot \varphi \mathrm{d} x\right|_{0} ^{\tau} \\
=\int_{0}^{\tau} \int_{\Omega}\left(\varrho \mathbf{u} \cdot \partial_{t} \varphi+\varrho(\mathbf{u}-\omega \times x) \otimes \mathbf{u}: \nabla_{x} \varphi+\overline{p(\varrho)} \operatorname{div}_{x} \varphi-\mathbb{S}\left(\nabla_{x} \mathbf{u}\right): \nabla_{x} \varphi\right) \mathrm{d} x \mathrm{~d} t
\end{gathered}
$$

for all $\tau \in[0, T]$ and for any $\varphi \in C_{c}^{1}\left([0, T] \times \Omega ; \mathbb{R}^{3}\right)$;

$$
\begin{aligned}
& \int_{0}^{\tau} \int_{\Omega} \overline{\varrho L_{k}(\varrho)}\left(\partial_{t} \varphi+(\mathbf{u}-\omega \times x) \cdot \nabla_{x} \varphi\right) \mathrm{d} x \mathrm{~d} t \\
& =\int_{0}^{\tau} \int_{\Omega} \overline{T_{k}(\varrho) \operatorname{div} \mathbf{u}} \varphi \mathrm{d} x \mathrm{~d} t+\left.\int_{\Omega} \overline{\varrho L_{k}(\varrho)} \varphi \mathrm{d} x\right|_{0} ^{\tau}
\end{aligned}
$$

and

$$
\int_{0}^{\tau} \int_{\Omega} \overline{T_{k}(\varrho)}\left(\partial_{t} \varphi+(\mathbf{u}-\omega \times x) \cdot \nabla_{x} \varphi\right) \mathrm{d} x \mathrm{~d} t
$$




$$
=\int_{0}^{\tau} \int_{\Omega} \overline{\left(\varrho T_{k}^{\prime}(\varrho)-T_{k}(\varrho)\right) \operatorname{div} \mathbf{u}} \varphi \mathrm{d} x \mathrm{~d} t+\left.\int_{\Omega} \overline{T_{k}(\varrho)} \varphi \mathrm{d} x\right|_{0} ^{\tau}
$$

where $\tau \in[0, T]$ and $\varphi \in C_{c}^{1}([0, T) \times \bar{\Omega})$. In the above formulas, we have taken

$$
T_{k}(z)=k T(z / k), L_{k}(z)=\int_{1}^{z} \frac{T_{k}(w)}{w^{2}} \mathrm{~d} w, \quad \forall z \geq 0, T(z)=\min \{z, k\} .
$$

\subsection{Strong convergence of densities}

\subsubsection{Effective viscous flux}

Using the same arguments as in [11, Chapter 3, Section 3.7] we can show that

$$
\overline{T_{k}(\varrho) \operatorname{div}_{x} \mathbf{u}}-\overline{T_{k}(\varrho)} \operatorname{div}_{x} \mathbf{u}=\frac{1}{\frac{4}{3} \mu+\eta}\left(\overline{p(\varrho) T_{k}(\varrho)}-\overline{p(\varrho)} \overline{T_{k}(\varrho)}\right) .
$$

To get this identity, one needs to subtract the limit $R \rightarrow \infty$ of the momentum equation (5.13) (written on $\Omega_{R}$ with $\left.\left(\varrho_{R}, \mathbf{u}_{R}\right)\right)$ tested with $\varphi=\zeta \nabla \Delta^{-1}\left[T_{k}\left(\varrho_{R}\right) \zeta\right]$, $\zeta \in C^{\infty}\left((0, T) \times \Omega_{n} ; \mathbb{R}^{3}\right)$ from the limiting momentum equation (6.11) with the test function $\varphi=\zeta \nabla \Delta^{-1}\left[\overline{T_{k}(\varrho)} \zeta\right]$, where $n$ is fixed. Here and hereafter we denote $\left(\nabla \Delta^{-1}\right)_{j}(v)=-\mathcal{F}^{-1}\left[\frac{\mathrm{i} \xi_{j}}{|\xi|^{2}} \mathcal{F}(v)(\xi)\right]$ (that is a continuous operator from $L^{p}\left(\mathbb{R}^{3}\right)$ to $\left\{\mathbf{v} \in L^{3 p /(3-p)}\left(\mathbb{R}^{3} ; \mathbb{R}^{3}\right), \quad \nabla \mathbf{v} \in L^{p}\left(\mathbb{R}^{3} ; \mathbb{R}^{3 \times 3}\right)\right\}, 1<p<3$ and $\mathcal{R}$ the Riesz operator, $(\mathcal{R}[v])_{i j}=\left(\nabla \otimes \nabla \Delta^{-1}\right)_{i j} v=\mathcal{F}^{-1}\left[\frac{\xi_{i} \xi_{j}}{|\xi|^{2}} \mathcal{F}(v)(\xi)\right]$ with $\mathcal{F}$ the Fourier transform. It is important to recall that $\mathcal{R}$ is a continuous operator from $L^{p}\left(\mathbb{R}^{3}\right) \rightarrow L^{p}\left(\mathbb{R}^{3}, \mathbb{R}^{3 \times 3}\right)$ for any $1<p<\infty$.

Since $\mathbb{S}$ is linear, this procedure yields

$$
\begin{gathered}
\lim _{R \rightarrow \infty} \int_{0}^{T} \int_{\Omega} \zeta(t, x)\left(p\left(\varrho_{R}\right) T_{k}\left(\varrho_{R}\right)-\mathbb{S}\left(\nabla_{x} \mathbf{u}_{R}\right): \mathcal{R}\left[\zeta T_{k}\left(\varrho_{R}\right)\right]\right) \mathrm{d} x \mathrm{~d} t \\
=\int_{0}^{T} \int_{\Omega} \zeta(t, x)\left(\overline{p(\varrho)} \overline{T_{k}(\varrho)}-\mathbb{S}\left(\nabla_{x} \mathbf{u}\right): \mathcal{R}\left[\zeta \overline{T_{k}(\varrho)}\right]\right) \mathrm{d} x \mathrm{~d} t \\
\lim _{R \rightarrow \infty} \int_{0}^{T} \int_{\Omega}\left(\mathbf{u}_{R}-\omega \times x\right) \cdot\left(\zeta T_{k}\left(\varrho_{R}\right) \mathcal{R}\left[\zeta \varrho_{R} \mathbf{u}_{R}\right]-\zeta \varrho_{R} \mathbf{u}_{R} \mathcal{R}\left[\zeta T_{k}\left(\varrho_{R}\right)\right]\right) \mathrm{d} x \mathrm{~d} t \\
-\int_{0}^{T} \int_{\Omega}\left(\mathbf{u}_{R}-\omega \times x\right) \cdot\left(\zeta \overline{T_{k}(\varrho)} \mathcal{R}[\zeta \varrho \mathbf{u}]-\zeta \varrho \mathbf{u} \mathcal{R}\left[\zeta \overline{T_{k}(\varrho)}\right]\right) \mathrm{d} x \mathrm{~d} t,
\end{gathered}
$$


where $(\mathcal{R}[\mathbf{z}])_{j}=\sum_{k=1}^{3} \mathcal{R}_{j k}\left[z_{k}\right]$. In fact, other (lower order) terms that should eventually appear at the right hand side are zero by virtue of a standard compactness argument.

At this stage, we need a div-curl type lemma in the form [11, Theorem 10.27], stating

Lemma 6.1. Let $\mathbf{U}_{R} \rightarrow \mathbf{U}$ in $L^{p}\left(\mathbb{R}^{3} ; \mathbb{R}^{3}\right), v_{R} \rightarrow v$ in $L^{q}\left(\mathbb{R}^{3}\right)$ as $R \rightarrow \infty$, where

$$
\frac{1}{p}+\frac{1}{q}=\frac{1}{r}<1 \text {. }
$$

Then

$$
v_{R} \mathcal{R}\left[\mathbf{U}_{R}\right]-\mathcal{R}\left[v_{R}\right] \mathbf{U}_{R} \rightarrow v \mathcal{R}[\mathbf{U}]-\mathcal{R}[v] \mathbf{U}
$$

in $L^{r}\left(\mathbb{R}^{3} ; \mathbb{R}^{3}\right)$.

Combining this lemma with the convergence established in the first two lines of (6.9), we get

$$
\left(\zeta T_{k}\left(\varrho_{R}\right) \mathcal{R}\left[\zeta \varrho_{R} \mathbf{u}_{R}\right]-\zeta \varrho_{R} \mathcal{R}\left[\zeta T_{k}\left(\varrho_{R}\right)\right] \mathbf{u}_{R}\right)(t) \rightarrow\left(\zeta \overline{T_{k}(\varrho)} \mathcal{R}[\zeta \varrho \mathbf{u}]-\zeta \varrho \mathcal{R}\left[\zeta \overline{T_{k}(\varrho)}\right] \mathbf{u}\right)(t)
$$

weakly in $L^{r}\left(\mathbb{R}^{3} ; \mathbb{R}^{3}\right)$ with some $r>6 / 5$ for all $t \in[0, T]$ as $R \rightarrow \infty$; whence by the compact imbedding $L^{r}\left(\Omega_{n}\right) \hookrightarrow \hookrightarrow W^{-1,2}\left(\Omega_{n}\right)$ and the Lebesgue dominated convergence theorem used over $(0, T)$ we conclude that the r.h.s. of $(6.15)$ is equal to 0 . This yields the effective viscous flux identity (6.14).

\subsubsection{Oscillations defect measure and renormalized continuity equation}

Due to $(2.2)$,

$$
p(\varrho)=d \varrho^{\gamma}+p_{m}(\varrho), \text { for some } d>0, \text { where } \partial_{\varrho} p_{m}(\varrho) \geq 0
$$

We have

$$
\begin{gathered}
d \limsup _{R \rightarrow 0} \int_{0}^{T} \int_{\Omega} \zeta\left|T_{k}\left(\varrho_{R}\right)-T_{k}(\varrho)\right|^{\gamma+1} \mathrm{~d} x \mathrm{~d} t \leq \\
d \limsup _{R \rightarrow \infty} \int_{0}^{T} \int_{\Omega} \zeta\left(\left(T_{k}\left(\varrho_{R}\right)-T_{k}(\varrho)\right)\left(\varrho_{R}^{\gamma}-\varrho^{\gamma}\right) \mathrm{d} x \mathrm{~d} t \leq\right. \\
\int_{0}^{T} \int_{\Omega} \zeta\left(\overline{\varrho^{\gamma} T_{k}(\varrho)}-\overline{\varrho^{\gamma}} \overline{T_{k}(\varrho)}\right) \mathrm{d} x \mathrm{~d} t \leq \int_{0}^{T} \int_{\Omega} \zeta\left(\overline{p(\varrho) T_{k}(\varrho)}-\overline{p(\varrho)} \overline{T_{k}(\varrho)}\right) \mathrm{d} x \mathrm{~d} t
\end{gathered}
$$

where $\zeta \in C_{c}^{\infty}((0, T) \times \Omega), \zeta \geq 0$. The first inequality is an algebraic one, to derive the second one, we have used convexity of $\varrho \rightarrow \varrho^{\gamma}$ and concavity of $\varrho \rightarrow T_{k}(\varrho)$. Finally, to 
derive the third one, we have employed the standard relation between the weak limits of monotone functions that reads

$$
\overline{p_{m}(\varrho) T_{k}(\varrho)}-\overline{p_{m}(\varrho)} \overline{T_{k}(\varrho)} \geq 0
$$

cf. e.g. [11, Theorem 10.19].

The right hand side of inequality (6.17) can be calculated from the effective viscous flux identity (6.14); using (6.6) we find that

$$
\begin{aligned}
& \int_{0}^{T} \int_{\Omega_{n}}\left|T_{k}\left(\varrho_{R}\right)-T_{k}(\varrho)\right|^{\gamma+1} \mathrm{~d} x \mathrm{~d} t \leq c(n)\left\|T_{k}\left(\varrho_{R}\right)-T_{k}(\varrho)\right\|_{L^{2}\left((0, T) \times \Omega_{n}\right)} \\
& \quad \leq\left\|T_{k}\left(\varrho_{R}\right)-T_{k}(\varrho)\right\|_{L^{1}\left((0, T) \times \Omega_{n}\right)}^{(\gamma-1) /(2 \gamma)}\left\|T_{k}\left(\varrho_{R}\right)-T_{k}(\varrho)\right\|_{L^{\gamma+1}\left((0, T) \times \Omega_{n}\right)}^{(\gamma+1) /(2 \gamma)}
\end{aligned}
$$

whence

$$
\text { osc }_{\gamma+1}\left[\varrho_{R} \rightarrow \varrho\right]\left((0, T) \times \Omega_{n}\right) \equiv \sup _{k>0} \limsup _{R \rightarrow \infty} \int_{0}^{T} \int_{\Omega_{n}}\left|T_{k}\left(\varrho_{R}\right)-T_{k}(\varrho)\right|^{\gamma+1} \mathrm{~d} x \mathrm{~d} t \leq c(n),
$$

where the expression at the left hand side is called oscillations defect measure, see Feireisl $[7]$.

On the other hand, relation (6.19) implies that the limit quantities $\varrho$, u satisfy the renormalized equation of continuity (2.5), see Feireisl [7],

Lemma 6.2 (Renormalized continuity equation). Let $G \subset \mathbb{R}^{3}$ be open set and let

$$
\begin{array}{cc}
\varrho_{R} \rightarrow \varrho & \text { in } L^{1}((0, T) \times G), \\
\mathbf{u}_{R} \rightarrow \mathbf{u} & \text { in } L^{r}\left((0, T) \times G ; \mathbb{R}^{3}\right), \\
\nabla \mathbf{u}_{R} \rightarrow \nabla \mathbf{u} & \text { in } L^{r}\left((0, T) \times G ; \mathbb{R}^{3 \times 3}\right), \quad r>1 .
\end{array}
$$

Let

$$
\operatorname{osc}_{q}\left[\varrho_{R} \rightarrow \varrho\right](G)<\infty
$$

for $\frac{1}{q}<1-\frac{1}{r}$, where $\left(\varrho_{R}, \mathbf{u}_{R}\right)$ solves the renormalized continuity equation (2.5) (with $\Omega$ replaced by $G$ ). Then the limit functions $\varrho$, $\mathbf{u}$ solve (2.5) with ( $\Omega$ replaced by $G$ ) as well.

\subsection{Strong convergence of density sequence}

We deduce using Lemma 6.2 that

$$
\int_{0}^{\tau} \int_{\Omega} \varrho L_{k}(\varrho)\left(\partial_{t} \varphi+(\mathbf{u}-\omega \times x) \cdot \nabla_{x} \varphi\right) \mathrm{d} x \mathrm{~d} t
$$




$$
=\int_{0}^{\tau} \int_{\Omega} T_{k}(\varrho) \operatorname{div} \mathbf{u} \varphi \mathrm{d} x \mathrm{~d} t+\left.\int_{\Omega} \varrho L_{k}(\varrho) \varphi \mathrm{d} x\right|_{0} ^{\tau}
$$

where $L_{k}(\varrho)$ is defined in (6.13), $\tau \in[0, T]$ and $\varphi \in C_{c}^{\infty}([0, T] \times \bar{\Omega})$. Reasoning as in (6.18) we verify that

$$
\overline{p(\varrho) T_{k}(\varrho)}-\overline{p(\varrho)} \overline{T_{k}(\varrho)} \geq 0 ;
$$

whence subtracting $(6.12),(6.21)$ and noticing that $(\overline{\varrho \log \varrho}-\varrho \log \varrho)(0)=0$, we deduce after letting $k \rightarrow \infty$,

$$
\begin{gathered}
\int_{\Omega}(\overline{\varrho \log \varrho}-\varrho \log \varrho)(\tau) \Phi_{R}(x) \\
\leq \int_{0}^{\tau} \int_{\Omega}\left(\overline{\varrho \log \varrho}-\varrho_{\infty} \log \varrho_{\infty}\right)(\mathbf{u}-\omega \times x) \cdot \nabla_{x} \Phi_{R}(x) \mathrm{d} x \mathrm{~d} t \\
-\int_{0}^{\tau} \int_{\Omega}\left(\varrho \log \varrho-\varrho_{\infty} \log \varrho_{\infty}\right)(\mathbf{u}-\omega \times x) \cdot \nabla_{x} \Phi_{R}(x) \mathrm{d} x \mathrm{~d} t .
\end{gathered}
$$

Here, we have used (6.22), and we have chosen $\Phi_{R}(x)=\eta\left(x_{3} / R^{\alpha}\right) \phi\left(\left|x^{\prime}\right| / R\right), R>1$, $\alpha>0, x^{\prime}=\left(x_{1}, x_{2}\right)$,

$$
\begin{gathered}
\eta \in C_{c}^{\infty}(\mathbb{R}), \phi \in C_{c}^{\infty}(\mathbb{R}), 0 \leq \eta, \phi \leq 1 \\
\phi\left(\left|x^{\prime}\right|\right)=\left\{\begin{array}{c}
\phi\left(\left|x^{\prime}\right|\right)=1 \text { if }\left|x^{\prime}\right| \leq 1 \\
\phi\left(\left|x^{\prime}\right|\right)=0 \text { if }\left|x^{\prime}\right| \geq 2
\end{array}\right\}, \eta(s)=\left\{\begin{array}{c}
\eta(s)=1 \text { if }|s| \leq 1 \\
\eta(s)=0 \text { if }|s| \geq 2
\end{array}\right\} .
\end{gathered}
$$

It is easy to check that

$$
\left\|\nabla_{x} \Phi_{R}\right\|_{L^{\infty}\left(R^{3} ; R^{3}\right)} \rightarrow 0 \text { as } R \rightarrow \infty,\left\|\partial_{x_{3}} \Phi_{R}\right\|_{L^{q}\left(R^{3}\right)}^{q} \leq c R^{2+\alpha-\alpha q}, 1 \leq q<\infty,
$$

and that

$$
(\omega \times x) \cdot \nabla_{x} \Phi_{R}(x)=0 .
$$

Consequently,

$$
\begin{gathered}
\int_{\Omega}(\overline{\varrho \log \varrho}-\varrho \log \varrho)(\tau) \Phi_{R}(x) \\
\leq \int_{0}^{\tau} \int_{\Omega}\left|\overline{\varrho \log \varrho}-\varrho_{\infty} \log \varrho_{\infty}\right|\left|\mathbf{U}_{\infty}\right|\left|\partial_{x_{3}} \Phi_{R}(x)\right| \mathrm{d} x \mathrm{~d} t \\
-\int_{0}^{\tau} \int_{\Omega}\left|\varrho \log \varrho-\varrho_{\infty} \log \varrho_{\infty}\right|\left|\mathbf{U}_{\infty}\right|\left|\partial_{x_{3}} \Phi_{R}(x)\right| \mathrm{d} x \mathrm{~d} t \\
+\int_{0}^{\tau} \int_{\Omega}\left(\varrho \log \varrho-\varrho_{\infty} \log \varrho_{\infty}\right)\left(\mathbf{u}-\mathbf{U}_{\infty}\right) \cdot \nabla_{x} \Phi_{R}(x) \mathrm{d} x \mathrm{~d} t
\end{gathered}
$$




$$
+\int_{0}^{\tau} \int_{\Omega}\left(\overline{\varrho \log \varrho}-\varrho_{\infty} \log \varrho_{\infty}\right)\left(\mathbf{u}-\mathbf{U}_{\infty}\right) \cdot \nabla_{x} \Phi_{R}(x) \mathrm{d} x \mathrm{~d} t
$$

Due to uniform bounds (6.2-6.6), the right hand side of this inequality tends to 0 as $R \rightarrow \infty$, provided we chose $\alpha>2$. Consequently,

$$
\overline{\varrho \log \varrho}-\varrho \log \varrho=0 ;
$$

whence

$$
\varrho_{R} \rightarrow \varrho \quad \text { a.e. in }(0, T) \times \Omega \text {. }
$$

\section{Remark 6.1}

It is to be noticed that the couple $(\varrho, \mathbf{u})$ satisfies the energy inequality in the form

$$
\begin{gathered}
\left.\int_{\Omega}\left(\frac{1}{2} \varrho\left|\mathbf{u}-\mathbf{U}_{\infty}\right|^{2}+E\left(\varrho \mid \varrho_{\infty}\right)\right) \mathrm{d} x\right|_{0} ^{\tau} \\
+\int_{0}^{\tau} \int_{\Omega}\left(\mathbb{S}\left(\nabla_{x}\left(\mathbf{u}-\mathbf{U}_{\infty}\right)\right): \nabla_{x}\left(\mathbf{u}-\mathbf{U}_{\infty}\right) \mathrm{d} x \mathrm{~d} t \leq\right. \\
-\int_{0}^{\tau} \int_{B_{1} \backslash S} p(\rho) \operatorname{div} \mathbf{U}_{\infty}-\int_{0}^{\tau} \int_{B_{1} \backslash S} \rho(\mathbf{u}-\omega \times x) \cdot \nabla_{x} \mathbf{U}_{\infty} \cdot\left(\mathbf{u}-\mathbf{U}_{\infty}\right) \mathrm{d} x \mathrm{~d} t \\
-\int_{0}^{\tau} \int_{B_{1} \backslash S} \varrho\left(\omega \times \mathbf{U}_{\infty}\right) \cdot\left(\mathbf{u}-\mathbf{U}_{\infty}\right) \mathrm{d} x \mathrm{~d} t-\int_{0}^{\tau} \int_{B_{1} \backslash S} \mathbb{S}\left(\nabla_{x} \mathbf{U}_{\infty}\right): \nabla_{x}\left(\mathbf{u}-\mathbf{U}_{\infty}\right) \mathrm{d} x \mathrm{~d} t
\end{gathered}
$$

for a. a. $\tau \in(0, T)$, where $\mathbf{U}_{\infty}$ is defined in (6.1).

\section{References}

[1] D. Bresch, B. Desjardins, D. Gérard-Varet. Rotating fluids in a cylinder, DCDS A, 11 (2004), 47-82.

[2] J.-Y. Chemin, B. Desjardins, I. Gallagher, and E. Grenier. Mathematical geophysics, volume 32 of Oxford Lecture Series in Mathematics and its Applications. The Clarendon Press Oxford University Press, Oxford, 2006.

[3] P. Deuring, S., Kračmar, Š. Nečasová. On pointwise decay of linearized stationary incompressible viscous flow around rotating and translating bodies, SIAM J. Math. Anal., 43 (2011), 705-738.

[4] R. Farwig. An $L^{q}$-analysis of viscous fluid flow past a rotating obstacle, Tôhoku Math. J. 920, 58 (2006), no.1, 129-147 


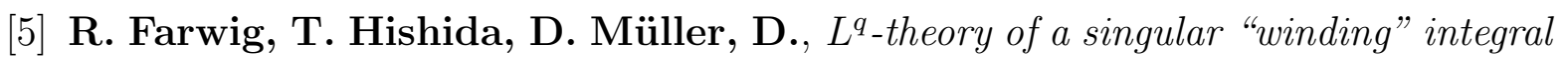
operator arising from fluid dynamics. Pacific J. Math. 215 (2004), 297-312

[6] R. Farwig, M. Krbec, Š. Nečasová., A weighted $L^{q}$ approach to Oseen flow around a rotating body, Math. Methods Appl.Sci. 31 (2008),5, 551-574

[7] E. Feireisl. Dynamics of viscous compressible fluids. Oxford University Press, Oxford, 2004.

[8] E. Feireisl. Low Mach number limits of compressible rotating fluids, J. Math. Fluid Mech. 14 (2012), no. 1, 618.

[9] E. Feireisl, I. Gallagher and A. Novotný. A singular limit for compressible rotating fluids. SIAM Journal on Mathematical Analysis 44 (1), 2012, 192-205.

[10] E. Feireisl, I. Gallagher, D. Gerard-Varet, A. Novotný ıMulti-scale Analysis of Compressible Viscous and Rotating Fluids Commun. Math. Phys. 314, 2012, 641-670.

[11] E. Feireisl and A. Novotný. Singular limits in thermodynamics of viscous fluids. Birkhäuser-Verlag, Basel, 2009.

[12] E. Feireisl, O. Kreml, Š. Nečasová, J. Neustupa, J. Stebel. Incompressible limits of fluids excited by moving boundaries, Submitted.

[13] E. Feireisl, O. Kreml, Š. Nečasová, J. Neustupa, and J. Stebel. Weak solutions to the barotropic NavierStokes system with slip boundary conditions in time dependent domains, J. Differential Equations, 254, (2013), 125-140, 2013.

[14] G.P. Galdi. On the motion of a rigid body in a viscous liquid: A mathematical analysis with applications, Handbook of Mathematical Fluid Dynamics, Vol. 1, 653791. Ed. by S. Friedlander, D. Serre, Elsevier 2002

[15] G. P. Galdi. An Introduction to the Mathematical Theory of the Navier-Stokes Equations. Steady - state problems, Springer-Verlag, New York-Berlin-Heidelberg, 2012

[16] G. P. Galdi, A. L. Silvestre. Further results on steady-state flow of a NavierStokes liquid around a rigid body. Existence of the wake, RIMS Kôkyûroku Bessatsu, B1 (2007), 108-127. 
[17] G. P. Galdi, M. Kyed. Steady-state Navier-Stokes flows past a rotating body: Leray solutions are physically reasonable, Arch. Rat. Mech. Anal., 200 (2011), 21-58.

[18] M. Geissert, H. Heck, M. Hieber., $L^{p}$ theory of the Navier-Stokes flow in the exterior of a moving or rotating obstacle, J. Reine Angew. Math. 596, 45-62 (2006)

[19] T. Hishida. An existence theorem for the Navier-Stokes flow in the exterior of a rotating obstacle, Arch. Rational Mech. Anal. 150, 307-348 (1999)

[20] D. Jesslé, B. J. Jin, A. Novotný. Navier-Stokes-Fourier system, weak solutions, relative entropy, weak-strong uniqueness, SIAM J. Math. Anal., to appear

[21] S. Kračmar, Š., Nečasová, P. Penel., Anisotropic L $L^{2}$-estimates of weak solutions to the stationary Oseen-type equations in 3D-exterior domain for a rotating body, J. Math. Soc. of Japan 62, 1, (2010) 239-268.

[22] J. Leray. Sur le mouvement d'un liquide visqueux emplissant l'espace. Acta Math., 63:193-248, 1934.

[23] P.-L. Lions. Mathematical topics in fluid dynamics, Vol.2, Compressible models. Oxford Science Publication, Oxford, 1998.

[24] S. Novo. The compressible Navier-Stokes equations with influx-outflux boundary conditions J. Math. fluid. Mech. (2003)

[25] A. Novotný, I. Straškraba. Introduction to the Mathematical Theory of Compressible Flow, Oxford University Press, Oxford, 2004.

[26] P. Sýkora Motion of a compressible fluid in a time dependent domain, Master thesis, Charles University of Prague, 2012 (in Czech). 